\title{
THE PULSATION MODE OF THE CEPHEID POLARIS
}

\author{
D. G. TURNeR ${ }^{1}$, V. V. KOVTYUKH ${ }^{2}$, I. A. USENKO ${ }^{2}$, AND N. I. GORLOVA ${ }^{3}$ \\ ${ }^{1}$ Department of Astronomy and Physics, Saint Mary's University, Halifax NS B3H 3C3, Canada; turner@ap.smu.ca \\ 2 Astronomical Observatory, Odessa National University, and Isaac Newton Institute of Chile, Odessa Branch, T. G. Shevkenko Park, 65014 Odessa, Ukraine \\ ${ }^{3}$ Institute of Astronomy, Celestijnenlaan 200D, B-3001 Leuven, Belgium \\ Received 2012 September 20; accepted 2012 November 23; published 2012 December 12
}

\begin{abstract}
A previously derived photometric parallax of $10.10 \pm 0.20$ mas, $d=99 \pm 2 \mathrm{pc}$, is confirmed for Polaris by a spectroscopic parallax derived using line ratios in high dispersion spectra for the Cepheid. The resulting estimates for the mean luminosity of $\left\langle M_{V}\right\rangle=-3.07 \pm 0.01$ s.e., average effective temperature of $\left\langle T_{\text {eff }}\right\rangle=6025 \pm 1 \mathrm{~K}$ s.e., and intrinsic color of $(\langle B\rangle-\langle V\rangle)_{0}=+0.56 \pm 0.01$ s.e., which match values obtained previously from the photometric parallax for a space reddening of $E_{B-V}=0.02 \pm 0.01$, are consistent with fundamental mode pulsation for Polaris and a first crossing of the instability strip, as also argued by its rapid rate of period increase. The systematically smaller Hipparcos parallax for Polaris appears discrepant by comparison.
\end{abstract}

Key words: stars: distances - stars: fundamental parameters - stars: individual (Polaris) - stars: variables: Cepheids

\section{INTRODUCTION}

Our current knowledge of the intrinsic properties of classical Cepheid variables relies heavily on the observational parameters derived for them. In the case of the nearest Cepheid, Polaris ( $\alpha$ UMi, $P=3.969$ days; see Turner et al. 2005), the distance and reddening are of paramount importance for understanding its pulsation mode and evolutionary status. A well-defined space reddening of $E_{B-V}=0.02 \pm 0.01$ is implied by studies of its optical companion and other stars lying in the immediate vicinity of the Cepheid (Turner 1977, 2006, 2009; Gauthier \& Fernie 1978), but the distance remains a point of contention.

A photometric parallax can be inferred for Polaris using its $18^{\prime \prime}$ distant F3 V companion (Turner 1977), which is recognized to be physically associated with the Cepheid on the basis of common proper motions and radial velocities (Kamper 1996). The distance derived from zero-age main-sequence (ZAMS) fitting for Polaris B is $101 \pm 3 \mathrm{pc}$ (Turner 2006), or $109.5 \mathrm{pc}$ from a spectroscopic investigation (Usenko \& Klochkova 2008). An investigation of spatially adjacent A-, F-, and G-type dwarfs within $3^{\circ}$ of Polaris observed by Hipparcos (ESA 1997) reveals two distinct groups lying along the line of sight (Turner 2004), of which only the closer contains stars of comparable proper motion and radial velocity to the Cepheid. The stars also concentrate spatially toward Polaris, and appear to constitute the remains of a sparse cluster in the final stages of dissolution into the Galactic disk. The implied distance from ZAMS fitting is $99 \pm 2 \mathrm{pc}$ (Turner 2006, 2009), and the corresponding photometric parallax is $10.10 \pm 0.20$ mas.

With the above reddening, the photometric distance implies $\left\langle M_{V}\right\rangle=-3.07 \pm 0.04$ (Turner 2006), consistent with fundamental mode pulsation for a 3.969 day Cepheid, along with a location near the center of the instability strip (Turner et al. 2005, 2006; Turner 2009). A potential conflict with the Cepheid's small light amplitude, more typical of stars on the hot and cool edges of the strip, was resolved by Turner $(2006,2009)$ using the observation that Polaris appears to be in the first crossing of the instability strip. The Cepheid's implied location near the strip center, despite an extremely low amplitude, can be attributed to a narrow and blueward-skewed instability strip for first crossers, in which surface convection damps pulsation at significantly warmer effective temperatures than for other crossings (see Alibert et al. 1999). The redward evolution of Polaris toward the cool edge of the instability strip for first crossers implied by its steady period increase (Turner et al. 2005; Turner 2009) is also consistent with its apparently decreasing light amplitude (Turner 2009).

Results for the trigonometric parallax of Polaris differ from those implied by its photometric parallax. Refractor parallaxes summarized by van Altena et al. (1995) yield a parallax of $4.0 \pm 3.3$ mas for Polaris, but that does not appear to account for a magnitude dependence in the original Allegheny parallaxes, attributed to the use of a rotating sector to diminish the flux from bright stars (Hanson 1978). If the magnitude-dependent correction found by Hanson \& Lutz (1983) is applied, the older parallaxes summarized by Jenkins $(1952,1963)$, calibrated relative to Allegheny parallaxes (e.g., Wagman 1956), yield a best value of $11 \pm 4$ mas for the trigonometric parallax of Polaris, in agreement with the photometric result.

In contrast, the Hipparcos parallax of $7.56 \pm 0.48$ mas (ESA 1997), or $7.54 \pm 0.11$ mas from the new reduction (van Leeuwen 2007), implies a distance of $133 \pm 2 \mathrm{pc}$ to Polaris. At that distance with the reddening cited previously, the luminosity of the Cepheid is $\left\langle M_{V}\right\rangle=-3.62 \pm 0.05$, which implies overtone pulsation, consistent with its sinusoidal light curve and small amplitude. But the intrinsic color of $(\langle B\rangle-\langle V\rangle)_{0}=$ $+0.56 \pm 0.01$ still leaves Polaris well inside the hot edge of the instability strip, and its rapid rate of period increase implies either a first or higher than third crossing of the instability strip, with an expected increasing light amplitude. This conflicts with the results for other small amplitude Cepheids as well as the long-term decreasing light amplitude observed prior to the 1965 "glitch" (Turner 2009). In addition, the A-, F-, and G-type dwarfs in the vicinity of Polaris lying at distances comparable to that inferred from the Hipparcos parallax do not share the proper motion or radial velocity of the Cepheid (Turner 2004), producing further inconsistencies.

Such contradictions were overlooked when Feast \& Catchpole (1997) and van Leeuwen et al. (2008) used Polaris as an overtone pulsator to calibrate the Cepheid period-luminosity relation using Hipparcos parallaxes. The Hipparcos parallax was also adopted by Weilen et al. (2000) and Evans et al. (2008) 
in their analyses of the orbit of the F6 V radial velocity companion, Polaris Ab, as well as by Nordgren et al. (2012) with the star's measured angular diameter to estimate its radius. The process can be inverted for the angular diameter, however, and in combination with the well-established period-radius relation for classical Cepheids (Turner \& Burke 2002; Turner et al. $2010)$ yields distance estimates of $93 \pm 2 \mathrm{pc}\left(\theta_{\mathrm{LD}}\right)$ and $97 \pm 2 \mathrm{pc}$ $\left(\theta_{\mathrm{UD}}\right)$ for fundamental mode pulsation, and $122 \pm 3 \mathrm{pc}\left(\theta_{\mathrm{LD}}\right)$ and $128 \pm 3 \mathrm{pc}\left(\theta_{\mathrm{UD}}\right)$ for overtone pulsation, where $\theta_{\mathrm{LD}}$ and $\theta_{\mathrm{UD}}$ apply to the limb-darkened and uniform disk solutions, respectively. Agreement with the results from the parallax solutions is less than ideal. Orbital radial velocity residuals (Lee et al. 2008) and tests of possible parameters for close orbital companions to Polaris (Turner 2009) also suggest the possibility of an extra star in the Polaris A subsystem, so an independent test of the distance, luminosity, and pulsation mode of the Cepheid would be useful.

Spectroscopic parallaxes can be derived for Cepheids using line ratios, the basis of temperature and luminosity $(\log g$ ) discrimination in Morgan and Keenan spectral classification (Gray \& Corbally 2009). Kovtyukh and his collaborators (Kovtyukh 2007; Kovtyukh et al. 2010, 2012a, 2012b) have taken the technique to its natural limits by using high dispersion spectra and all possible line ratios, in conjunction with calibrations on $T_{\text {eff }}$ and $M_{V}$, to establish the luminosities and effective temperatures of yellow supergiants and Cepheids with very high precision. Details of the calibration philosophy using line ratios involving primarily iron peak elements in the spectra of supergiants of well-established luminosity are provided by Kovtyukh \& Chekhonadskikh (2009). Typically, the precision averages \pm 0.26 in absolute visual magnitude $\left(M_{V}\right)$ for a single line ratio in Cepheids, but with so many line ratios available per spectrum, of the order of 40-70 (Kovtyukh et al. 2012b), the precision reached per spectrum reaches a few hundredths of a magnitude.

The method, although calibrated using yellow supergiants, was established with the aim of studying Cepheid variables, and was used recently by Kovtyukh et al. (2012b) to examine the pulsation modes of three s-Cepheids, with fairly robust results: V1334 Cyg (first overtone), V440 Per (fundamental mode), and V636 Cas (fundamental mode). It is ideal for learning more about Polaris, another s-Cepheid of extremely small amplitude and an object that is bright and readily accessible from northern hemisphere sites. The present study addresses the ongoing problem of the distance and pulsation mode of Polaris using its spectroscopic parallax.

\section{OBSERVATIONS AND DATA REDUCTIONS}

Observations of Polaris were obtained using the $6 \mathrm{~m}$ Large Azimuth Telescope (BTA) of the Special Astrophysical Observatory of the Russian Academy of Sciences equipped with the Nasmyth Echelle Spectrometer (Panchuk et al. 2009), which has a resolving power of $R \approx 60,000$ within the wavelength range 4380-6690 $\AA$. The signal-to-noise ratio at the continuum level in each of the 20-27 spectral orders exceeds 70. A thorium-argon lamp was used for wavelength calibration, and the data reduction was carried out using the MIDAS software ECHELLE modified for extraction of echelle spectra obtained with an image slicer (Yushkin \& Klochkova 2005). The spectra were extracted from the CCD images in the usual fashion: bias subtraction, flat fielding, cosmic-ray removal, and wavelength calibration.
Table 1

Spectroscopic Results for Polaris

\begin{tabular}{lccrrrrrrr}
\hline \hline JD (obs) & Phase & $\begin{array}{r}T_{\text {eff }} \\
\text { (K) }\end{array}$ & $\begin{array}{r}\text { s.d. } \\
(\mathrm{K})\end{array}$ & & $\begin{array}{r}\text { s.e. } \\
(\mathrm{K})\end{array}$ & $M_{V}$ & s.d. & $n$ & s.e. \\
\hline 2452861.5600 & 0.6293 & 6033 & 59 & 61 & 7.5 & -3.03 & 0.20 & 55 & 0.03 \\
2452867.5620 & 0.1402 & 6009 & 35 & 57 & 4.6 & -2.98 & 0.17 & 52 & 0.02 \\
2452869.5700 & 0.6456 & 5996 & 61 & 62 & 7.7 & -3.02 & 0.24 & 44 & 0.04 \\
2453072.1650 & 0.6452 & 6015 & 74 & 56 & 9.9 & -3.05 & 0.27 & 67 & 0.03 \\
2453073.6220 & 0.0120 & 6013 & 88 & 56 & 11.8 & -3.06 & 0.21 & 66 & 0.03 \\
2453162.1910 & 0.3075 & 6050 & 61 & 57 & 8.1 & -3.05 & 0.25 & 67 & 0.03 \\
2453689.6470 & 0.0829 & 6050 & 49 & 74 & 5.6 & -3.10 & 0.33 & 62 & 0.04 \\
2453690.1090 & 0.1992 & 6034 & 42 & 74 & 4.9 & -3.06 & 0.23 & 62 & 0.03 \\
2453693.1240 & 0.9582 & 6044 & 61 & 72 & 7.2 & -3.01 & 0.34 & 68 & 0.04 \\
2453980.5890 & 0.3201 & 6018 & 41 & 73 & 4.8 & -3.04 & 0.16 & 62 & 0.02 \\
2454073.5890 & 0.7303 & 6051 & 46 & 55 & 6.2 & -3.04 & 0.12 & 53 & 0.02 \\
2454077.6510 & 0.7528 & 6093 & 125 & 5 & 55.9 & -3.17 & 0.15 & 8 & 0.05 \\
2454169.6380 & 0.9080 & 6081 & 97 & 6 & 39.4 & -3.03 & 0.13 & 10 & 0.04 \\
2454225.2280 & 0.9012 & 6069 & 27 & 57 & 3.6 & -3.02 & 0.15 & 60 & 0.02 \\
2454345.5510 & 0.1889 & 6073 & 35 & 62 & 4.4 & -3.07 & 0.16 & 61 & 0.02 \\
2454426.0180 & 0.4440 & 5993 & 47 & 71 & 5.6 & -3.11 & 0.23 & 65 & 0.03 \\
2454934.5880 & 0.4598 & 6005 & 52 & 63 & 6.5 & -3.01 & 0.22 & 60 & 0.03 \\
2455005.3720 & 0.2772 & 5982 & 45 & 74 & 5.2 & -3.14 & 0.20 & 65 & 0.03 \\
2455324.6730 & 0.6493 & 5998 & 47 & 64 & 5.9 & -3.11 & 0.15 & 70 & 0.02 \\
2455328.5976 & 0.6372 & 5990 & 41 & 66 & 5.1 & -3.09 & 0.19 & 64 & 0.02 \\
2455708.3364 & 0.2212 & 5996 & 52 & 61 & 6.6 & -3.06 & 0.21 & 69 & 0.03 \\
2455816.5457 & 0.4583 & 5959 & 67 & 75 & 7.7 & -3.10 & 0.20 & 63 & 0.03 \\
2455901.6061 & 0.8686 & 6017 & 79 & 74 & 9.2 & -3.18 & 0.17 & 62 & 0.02 \\
\hline & & & & & & & & & \\
\hline
\end{tabular}

Spectra were also obtained with the fiber echelle-type spectrograph HERMES, mounted on the $1.2 \mathrm{~m}$ Belgian telescope on La Palma. A high-resolution configuration with $R=85,000$ for the wavelength region $3800-9000 \AA$ was used. The spectra were reduced using a Python-based pipeline that includes order extraction, wavelength calibration with $\mathrm{Th}-\mathrm{Ne}-\mathrm{Ar}$ arcs, flat-field division, cosmic-ray clipping, and order merging. Further details of the spectrograph and pipeline are given by Raskin et al. (2011). The dates of observation are indicated in Table 1.

\section{RESULTS}

The results of the analysis are summarized in Table 1 and plotted as a function of pulsation phase in Figure 1, where the phases were calculated with the regular period increase removed (Turner et al. 2005; Turner 2009). The plotted gray bands are not least-squares solutions to the data. They represent the values with their cited uncertainties determined for Polaris by Turner (2006) from the photometric parallax in conjunction with the reddening inferred from its companion. The observations themselves are fairly randomly distributed in phase around the Cepheid's cycle, and yield weighted mean values of $\left\langle M_{V}\right\rangle=-3.07 \pm 0.01$ s.e., and $\left\langle T_{\text {eff }}\right\rangle=6025 \pm 1 \mathrm{~K}$ s.e., identical to predictions (Turner 2006). They confirm the previous conclusion that Polaris is a fundamental mode pulsator. If it were pulsating in an overtone mode at the distance implied by its Hipparcos parallax, the observational data would yield significantly greater luminosities closer to $M_{V} \simeq-3.6$.

Curves in Figure 1 represent the expected sinusoidal variations in visual light and a Fourier fit to the effective temperature estimates. The data are precise enough to track pulsational changes in the latter. The star reaches its highest effective temperature two-tenths of a cycle prior to maximum light near minimum radius, and its lowest effective temperature seven-tenths of a cycle later near maximum radius. The temperature variations are skewed, unlike the more sinusoidal light variations, 


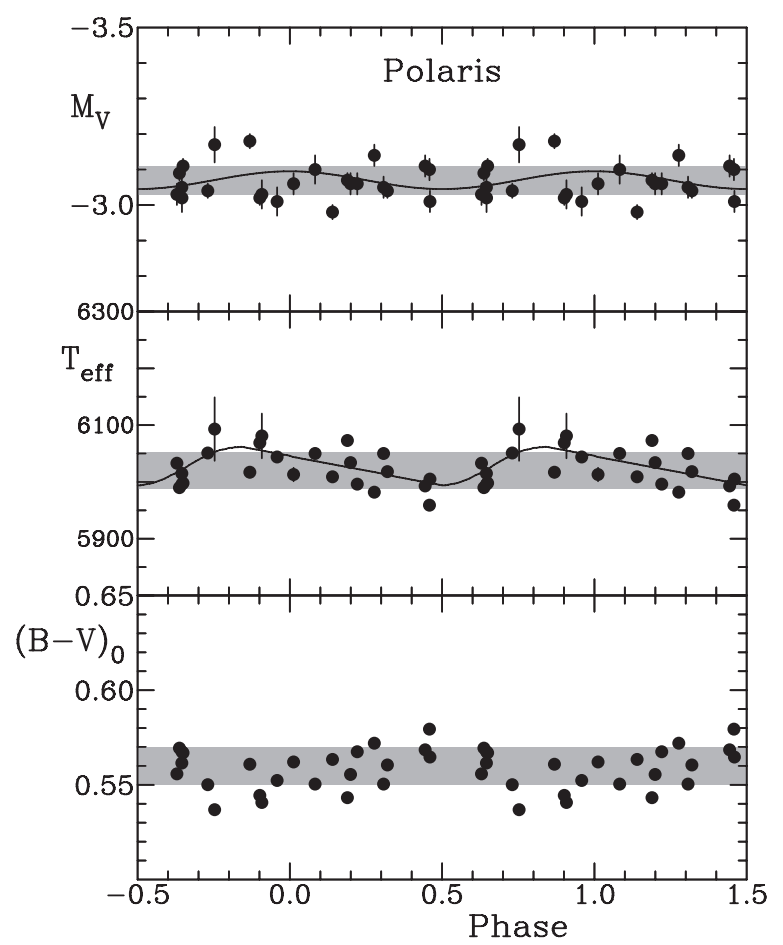

Figure 1. Luminosity (top), $T_{\text {eff }}$ (middle), and predicted color variations (bottom) for Polaris from the spectra analyzed here, with uncertainties in the data indicated. The gray bands represent the range of values predicted for Polaris from its photometric parallax and space reddening (Turner 2006), with superposed curves representing the light variations typical of the era of observation and a Fourier fit to the $T_{\text {eff }}$ data.

with a variation of $\sim 75 \mathrm{~K}$ in temperature over the course of a cycle. The color variations predicted by the estimates of effective temperature (see Turner \& Burke 2002; Turner et al. 2010) mimic the temperature changes in displaying skewness, and yield an unweighted mean value of $(\langle B\rangle-\langle V\rangle)_{0}=+0.56 \pm 0.01$ s.e. for Polaris. By comparison, the changes in absolute magnitude appear to be relatively small and sinusoidal, as observed for the visual light variations. Although the predicted and actual changes are similar, scatter in the individual estimates hinders more definitive conclusions. The trends are otherwise as expected for a fundamental mode pulsator.

The abundance patterns in Polaris- $[\mathrm{C} / \mathrm{H}]=-0.17$, $[\mathrm{N} / \mathrm{H}]=+0.42,[\mathrm{O} / \mathrm{H}]=-0.00$, and $[\mathrm{Na} / \mathrm{H}]=+0.09$ (Usenko et al. 2005) - are those of a star displaying the products of core CNO processing at its surface. Sometimes that is taken to be a signature that a Cepheid has passed through the red supergiant stage, where deep envelope convective dredge-up is thought to bring core-processed material to the stellar surface (Mowlavi 1999a, 1999b). But some stellar evolutionary models do not involve a dredge-up stage for red supergiants (e.g., Bono et al. 2000), and Maeder (2001) has noted that most B-type stars in late main-sequence hydrogen-burning stages prior to evolution toward the Cepheid instability strip already displayed CNOprocessed material in their atmospheres. Rapid rotation during main-sequence stages in conjunction with meridional mixing of core material to the stellar surface is sufficient to explain the abundance patterns in Cepheids (see Turner \& Berdnikov 2004), and that may be the case for Polaris, i.e., its progenitor was a rapid rotator as a B-dwarf. The atmospheric abundances of Polaris A and B (Usenko et al. 2005; Usenko \& Klochkova 2008) are otherwise indicative of slightly metal-rich stars with $[\mathrm{Fe} / \mathrm{H}]=+0.07$.

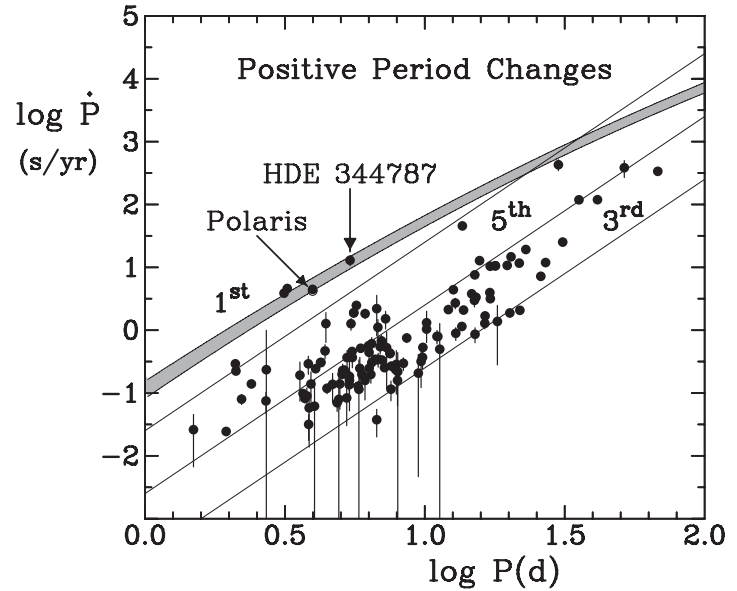

Figure 2. Observed and predicted rates of period increase for Cepheids in the first, third, and putative fifth crossings of the instability strip, i.e., Cepheids exhibiting period increases. Lines separate what appear to be stars in the third and fifth crossings, and a gray band represents predictions for first-crossing Cepheids according to stellar evolutionary models (see the text). The small amplitude Cepheids Polaris and HDE 344787 display period increases expected for a first crossing.

\section{POLARIS AS A FIRST-CROSSING CEPHEID}

A potential problem with the spectroscopic parallax concerns the conclusion reached previously that Polaris is in the first crossing of the instability strip, as inferred from its observed rate of period increase of $\sim 4.5 \mathrm{~s} \mathrm{yr}^{-1}$ (Turner et al. 2005; Turner 2009). Neilson et al. (2012) argue that the value appears smaller than what is predicted by stellar evolutionary models (Turner et al. 2006), and propose a higher strip crossing mode for Polaris that includes a component of the Cepheid's period increase arising from mass loss.

There is an alternative solution. In their comparison of observed rates of period change in Cepheids with those predicted from stellar evolutionary models, Turner et al. (2006) employed a semi-empirical approach (Turner 1996; Turner \& Burke 2002) in which Cepheid radii varied as $P^{0.75}$ and masses as $P^{0.5}$, where $P$ is the pulsation period. That yielded an equation for rate of period change of

$$
\frac{\dot{P}}{P}=\frac{6}{7} \frac{\dot{L}}{L}-\frac{24}{7} \frac{\dot{T}}{T},
$$

where the quantities on the right-hand side of the equation are taken from evolutionary models for stars crossing the Cepheid instability strip.

More recent studies of Cepheids belonging to open clusters indicate that the $P^{0.5}$ dependence for Cepheid masses is too steep, and more likely varies as $P^{0.4}$ (Turner 2012). With that adjustment the predicted rates of period change are modified to

$$
\frac{\dot{P}}{P}=\frac{5}{8} \frac{\dot{L}}{L}-\frac{5}{2} \frac{\dot{T}}{T}
$$

The effect is to reduce the predicted rates of period change for Cepheids in the first crossing of the instability strip, as depicted in Figure 2. The observed rate of period increase for Polaris is now fully consistent with a first crossing of the instability strip, without the need to postulate mass loss or overtone pulsation for a different strip crossing. The observed rate falls so close to the minimum predicted rate, in fact, that mass loss in the Cepheid must be almost negligible. 


\section{SUMMARY}

An independent test of the phase-dependent variations in luminosity and effective temperature of Polaris is made using line ratios in high dispersion spectra for the star, calibrated using stars of similar metallicity to the Cepheid. The observational results are relevant to the star's distance and pulsation mode. The derived absolute magnitudes $M_{V}$ and effective temperatures $T_{\text {eff }}$ coincide exactly with similar parameters predicted from the star's photometric parallax (Turner 2006). The spectroscopic and photometric parallaxes both imply a distance to Polaris of $99 \pm 2$ pc. The associated Hipparcos parallax for Polaris (ESA 1997; van Leeuwen 2007) appears to be discrepant by comparison.

The results are consistent with fundamental mode pulsation in Polaris, as well as with a first crossing of the instability strip. A correction of previously published predictions for first-crossing Cepheids (Turner et al. 2006) to account for a more correct period-mass relation for Cepheids brings the observed rate of period increase in Polaris into good agreement with predictions from stellar evolutionary models.

Some of the spectra were collected with the Mercator Telescope, operated on the island of La Palma by the Flemish Community, at the Spanish Observatorio del Roque de los Muchachos of the Instituto de Astrofisica de Canarias. We are greatful to Drs. V. G. Klochkova and M. V. Yushkin for obtaining the BTA spectra, and to Richard Anderson and Lovro Palaversa for allowing us to use their Mercator spectra prior to publication. Much of the information about the supergiants used in the calibration was gathered with the help of SIMBAD. We are grateful to the referee for useful suggestions on the presentation of the Letter.

\section{REFERENCES}

Alibert, Y., Baraffe, I., Hauschildt, P., \& Allard, F. 1999, A\&A, 344, 551 Bono, G., Caputo, F., Cassisi, S., et al. 2000, ApJ, 543, 955

ESA 1997, The Hipparcos and Tycho Catalogues, European Space AgencySP1200 (Noordwijk, Netherlands: ESA Publications Division)

Evans, N. R., Schaefer, G. H., Bond, H. F., et al. 2008, ApJ, 136, 1137

Feast, M. W., \& Catchpole, R. M. 1997, MNRAS, 286, L1

Gauthier, R. P., \& Fernie, J. D. 1978, PASP, 90, 739

Gray, R. O., \& Corbally, C. J. 2009, Stellar Spectral Classification (Princeton, NJ: Princeton Univ. Press)

Hanson, R. B. 1978, in IAU Colloq. 48, Modern Astrometry, ed. F. V. Prochazha \& R. H. Tucker (Vienna, Austria: Vienna Univ. Obs.), 31
Hanson, R. B., \& Lutz, T. E. 1983, MNRAS, 202, 201

Jenkins, L. F. 1952, General Catalogue of Trigonometric Stellar Parallaxes (New Haven, CT: Yale Univ. Obs.)

Jenkins, L. F. 1963, Supplement to the General Catalogue of Trigonometric Stellar Parallaxes (New Haven, CT: Yale Univ. Obs.)

Kamper, K. W. 1996, JRASC, 90, 140

Kovtyukh, V. V. 2007, MNRAS, 378, 617

Kovtyukh, V. V., \& Chekhonadskikh, F. A. 2009, in Proc. 16th Young Scientists' Conference on Astronomy and Space Physics, ed. V. Ya. Choliy \& G. Ivashchenko (Kiev, Ukraine: Kyivskyi Univ.), 28

Kovtyukh, V. V., Chekhonadskikh, F. A., Luck, R. E., et al. 2010, MNRAS, 408, 1568

Kovtyukh, V. V., Gorlova, N. I., \& Belik, S. A. 2012a, MNRAS, 423, 3268

Kovtyukh, V. V., Luck, R. E., Chekhonadskikh, F. A., \& Belik, S. A. 2012b, MNRAS, 426, 398

Lee, B.-C., Mkrtichian, D. E., Han, I., Park, M.-G., \& Kim, K.-M. 2008, AJ, 135,2240

Maeder, A. 2001, Ap\&SS, 277, 291

Mowlavi, N. 1999a, A\&A, 344, 617

Mowlavi, N. 1999b, A\&A, 350, 73

Neilson, H. R., Engle, S. G., Guinan, E., et al. 2012, ApJ, 745, L32

Nordgren, T. E., Armstrong, J. T., Germain, M. E., et al. 2000, ApJ, 543, 972

Panchuk, V. E., Klochkova, V. G., Yushkin, M. V., \& Najdenov, I. D. 2009, JOT, 76,87

Raskin, G., van Winckel, H., Hensberge, H., et al. 2011, A\&A, 526, 69

Turner, D. G. 1977, PASP, 89, 550

Turner, D. G. 1996, JRASC, 90, 82

Turner, D. G. 2004, BAAS, 36, 744

Turner, D. G. 2006, OAP, 18, 115

Turner, D. G. 2009, in AIP Conf. Proc. 1170, Stellar Pulsation: Challenges for Theory and Observation, ed. J. A. Guzik \& P. A. Bradley (Melville, NY: AIP), 59

Turner, D. G. 2012, JAVSO, 40, 502

Turner, D. G., Abdel-Sabour Abdel-Latif, M., \& Berdnikov, L. N. 2006, PASP, 118,410

Turner, D. G., \& Berdnikov, L. N. 2004, A\&A, 423, 335

Turner, D. G., \& Burke, J. F. 2002, AJ, 124, 2931

Turner, D. G., Majaess, D. J., Lane, D. J., et al. 2010, OAP, 23, 119

Turner, D. G., Savoy, J., Derrah, J., Abdel-Sabour Abdel-Latif, M., \& Berdnikov, L. N. 2005, PASP, 117, 207

Usenko, I. A., \& Klochkova, V. G. 2008, MNRAS, 387, L1

Usenko, I. A., Miroshnichenko, A. S., Klochkova, V. G., \& Yushkin, M. V. 2005, MNRAS, 362, 1219

van Altena, W. F., Lee, J. T., \& Hoffleit, E. D. 1995, The General Catalogue of Trigonometric Stellar Parallaxes (4th ed.; New Haven, CT: Yale Univ. Obs.) van Leeuwen, F. 2007, A\&A, 474, 653

van Leeuwen, F., Feast, M. W., Whitelock, P. A., \& Laney, C. D. 2008, MNRAS, 379,723

Wagman, N. E. 1956, PAO, 10, 32

Weilen, R., Jahreiss, B., Dettburn, C., Lenhardt, H., \& Schwan, H. 2000, A\&A, 360,390

Yushkin, M. V., \& Klochkova, V. G. 2005, Preprint, Special Astrophysical Observatory of the Russian Academy of Sciences, No. 206 